\title{
On the Steady-State System Size Distribution for a Discrete-Time Geo/G/1 Repairable Queue
}

\author{
Renbin Liu and Zhaohui Deng \\ School of Mathematics and Statistics, Chongqing University of Technology, Chongqing 400054, China \\ Correspondence should be addressed to Renbin Liu; liurb888@126.com
}

Received 28 September 2013; Accepted 10 February 2014; Published 19 March 2014

Academic Editor: Carlo Piccardi

Copyright (C) 2014 R. Liu and Z. Deng. This is an open access article distributed under the Creative Commons Attribution License, which permits unrestricted use, distribution, and reproduction in any medium, provided the original work is properly cited.

\begin{abstract}
This paper studies a discrete-time N-policy Geo/G/1 queueing system with feedback and repairable server. With a probabilistic analysis method and renewal process theory, the steady-state system size distribution is derived. Further, the steady-state system size distribution derived in this work is extremely suitable for numerical calculations. Numerical example illustrates the important application of steady-state system size distribution in system capacity design for a network access proxy system.
\end{abstract}

\section{Introduction}

Over the past years, the analysis of discrete-time queueing systems has received more attention in the literatures. This is because the discrete-time queues are more appropriate than their continuous-time counterparts in their applicability for many computer and communication systems in which time is divided into fixed-length time intervals (for details, e.g., see [1-4]).

It is well known that the N-policy introduced by Yadin and Naor [5] is very important in the theory and applications of queueing models. For detailed overviews of the continuous-time queues with N-policy, the reader is referred to an excellent survey by Tadj and Choudhury [6]. Related works on the discrete-time queues with $\mathrm{N}$-policy can be found in [7, 8]. Recently, Wei et al. [9] studied the transient and equilibrium properties of the queue length for an $\mathrm{N}$ policy Geo/G/1 queue with variable input rate and indicated that the stochastic decomposition property no longer holds. Wang [10] considered a random N-policy Geo/G/1 queue with start-up and closedown times and obtained analytic solutions of system size, lengths of state periods, and sojourn time. As far as known to the authors, very few papers about discrete-time $\mathrm{N}$-policy queueing systems are available.

Feedback was introduced by Takács [11] and since then many papers have appeared about this topic on continuoustime case (see, e.g., [12-14]). Atencia and Moreno [15] first extended the study to the discrete-time $\mathrm{Geo}^{\mathrm{X}} / \mathrm{G}_{H} / 1$ retrial queue. The phenomenon of feedback has many practical applications, for example, in telecommunication systems where messages that produce errors at the destination are sent again, in a call center where customers may call again (repeat their service) if their problems are not completely solved after the service and so forth. However, relative to continuous-time feedback queues, their discrete-time counterparts received very little attention in the literature.

A remarkable phenomenon in a queueing system is its server breakdown. For instance, in computer systems, the machine may be subject to scheduled backups and unforeseeable failures. Queues with server breakdowns can also be called repairable queues. For only the studies on discrete-time repairable queues, the reader may refer to Wang and Zhang [16], Tang et al. [17], and Liu and Gao [18].

However, only very few works in the literature concerned with $\mathrm{N}$-policy queues with feedback and repairable server have been done. Particularly, the researches of the discretetime $\mathrm{N}$-policy queues with feedback and repairable server are not found. Further, in most works of the N-policy queue, the probability generating function (PGF) of steadystate system size distribution rather than steady-state system size distribution was obtained. In this paper, we investigate a discrete-time N-policy Geo/G/1 queueing system with feedback and repairable server. With a probability analysis 
method and renewal process theory, we derive the steadystate system size distribution. It is noted that the steadystate system size distribution derived in this paper is quite suitable for numerical calculations. What is more, numerical examples illustrate the applications of steady-state system size distribution in system capacity design for a network access proxy system.

The rest of the paper is organized as follows. The next section presents the model assumptions and some preliminaries. In Section 3 we discuss the transient system size distribution and derive the steady-state system size distribution and its PGF. Section 4 numerically shows the important application of system size distribution in system capacity for a network access proxy system. Conclusions are finally drawn in Section 5 .

\section{Assumptions and Preliminaries}

We consider the following discrete-time N-policy Geo/G/1 queueing system with Bernoulli feedback and repairable server.

(1) Let the time axis be slotted into intervals of equal length with the length of a slot being one unit. To be more specific, let the time axis be marked by $0,1, \ldots, n, \ldots$ Here we discuss the model for a late arrival system with delayed access (LAS-DA) and therefore a potential arrival takes place in $\left(n^{-}, n\right)$ and a potential departure occurs in $\left(n, n^{+}\right)$; for details, see Hunter [19]. The various time epochs involved in our model can be viewed through a self-explanatory figure (see Figure 1). The interarrival times between customers, $\left\{\tau_{i}, i \geq\right.$ $1\}$, are independent identically distributed (i.i.d.) random variables with a geometric distribution $P\left\{\tau_{i}=j\right\}=p(1-$ $p)^{j-1},(j \geq 1,0<p<1)$.

(2) When there are $N(N \geq 1)$ customers present in system the server begins to serve in the first-come, firstserved (FCFS) order until no customer is present. The service times, $\left\{\chi_{n}, n \geq 1\right\}$, are i.i.d. random variables having distribution $g_{j}=P\left\{\chi_{n}=j\right\}, j \geq 1$, with $\operatorname{PGF} G(z)=$ $\sum_{j=1}^{\infty} g_{j} z^{j},|z|<1$, and mean $\mu$. The customer whose service has been just finished comes back to the queue with probability $1-\theta(0<\theta \leq 1)$ waiting for the next service or leaves forever with probability $\theta$.

(3) The server may fail when and only when it is serving a customer. The failed server will be repaired immediately. After repair, the server is as good as new and continues to serve the customer whose service has not been finished yet. We assume that the service time for a customer is cumulative.

(4) The lifetime of server has a geometric distribution with parameter $\alpha$, and its repair time $Y$ obeys an arbitrary distribution $y_{j}=P\{Y=j\}, j \geq 1$ with PGF $y(z)=\sum_{j=1}^{\infty} y_{j} z^{j}$, $|z|<1$, and a mean repair time $\beta$.

(5) All random variables are mutually independent. At the initial time $0^{+}$, the server begins to serve when the number of customers present in the system $N\left(0^{+}\right)>0$ or the server is idle and waits for the first arrival when $N\left(0^{+}\right)=0$. After the first busy period, the server will take an N-policy.
Remark 1. Throughout this paper, we adopt the following notations. $E(X)$ is the mean of random variable $X . P(Q)$ is the probability of event $Q . N\left(n^{+}\right)$denotes the system size at epoch $n^{+} . C_{l}^{k}=l ! / k !(l-k) !, 0 \leq k \leq l \cdot \bar{p}=1-p \cdot f(z)=p z /(1-\bar{p} z)$, $|z|<1$.

Definition 2. The "generalized service time of a customer" $\tilde{\chi}$ denotes the time interval from the time when the server begins to serve a customer until the service of this customer ends, which includes some possible repair times of the server due to its failures in the process of serving this customer.

Lemma 3 (see [17]). The PGF and the mean of $\tilde{\chi}$ are given, respectively, by

$$
\begin{aligned}
& \widetilde{G}(z)=\sum_{j=1}^{\infty} P(\tilde{\chi}=j) z^{j}=G[z \alpha y(z)+z(1-\alpha)], \quad|z|<1, \\
& E(\tilde{\chi})=\left.\frac{d \widetilde{G}(z)}{d z}\right|_{z=1}=\mu(1+\alpha \beta)
\end{aligned}
$$

where $G(z)$ and $y(z)$ are given by assumptions (2) and (4).

For customer $n$, let $\Omega_{n}, \widehat{\chi}_{n}$, and $\tilde{\chi}_{n}^{\mathrm{i}}$ denote total generalized service number, total generalized service time, and the $i$ th generalized service time before departure, respectively. Then it follows from assumption (2) that $P\left(\Omega_{n}=k\right)=\theta(1-\theta)^{k-1}$, $k \geq 1$, and $\widehat{\chi}_{n}=\sum_{i=1}^{\Omega_{n}} \widetilde{\chi}_{n}^{i}, n \geq 1$, are i.i.d. random variables, where $\tilde{\chi}_{n}^{i}, i, n \geq 1$, are mutually independent with the same distribution as $\widetilde{\chi}_{n}, n \geq 1$.

Lemma 4. Let $\widehat{g}_{j}=P(\widehat{\chi}=j), j \geq 1$, be the distribution of total generalized service time $\hat{\chi}$ for one customer before departure. Then one gets

$$
\begin{aligned}
\widehat{g}_{j} & =P\left\{\sum_{i=1}^{\Omega_{n}} \tilde{\chi}_{n}^{i}=j\right\} \\
& =\sum_{k=1}^{j} \theta(1-\theta)^{k-1} P\left\{\sum_{i=1}^{k} \widetilde{\chi}_{n}^{i}=j\right\}, \quad j \geq 1, \\
\widehat{G}(z) & =\sum_{j=1}^{\infty} \widehat{g}_{j} z^{j}=\frac{\theta \widetilde{G}(z)}{1-(1-\theta) \widetilde{G}(z)}, \quad|z|<1, \\
E(\widehat{\chi}) & =\left.\frac{d \widehat{G}(z)}{d z}\right|_{z=1}=\frac{\mu(1+\alpha \beta)}{\theta},
\end{aligned}
$$

where $\widetilde{G}(z)$ is given by Lemma 3 .

Definition 5. The "server busy period" denotes the time interval from the time when the server begins to serve a customer until the system becomes empty, which contains some possible repair times of server due to its failures in the process of service. 


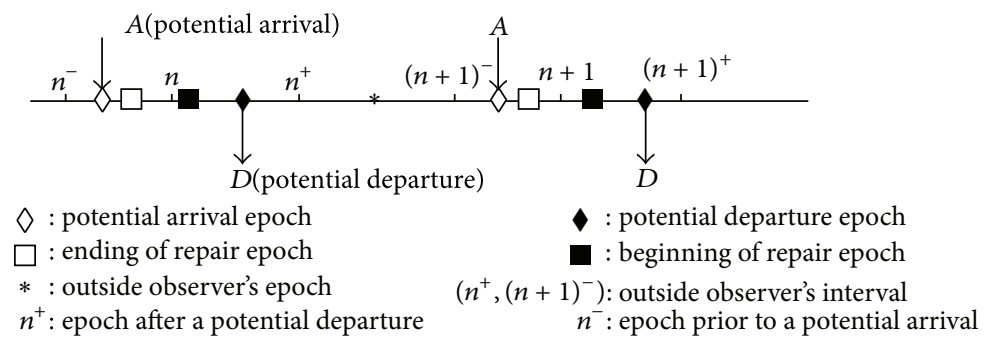

FIGURE 1: Various time epochs in a late arrival system with delayed access.

Let $b$ denote the server busy period initiated with one customer, and its PGF is $b(z)=\sum_{j=0}^{\infty} P(b=j) z^{j},|z|<1$. According to [20], the following lemma holds.

Lemma 6. $b(z)$ satisfies the equation $b(z)=\widehat{G}[(1-p) z+$ $p b(z) z]$, and

$$
E(b)= \begin{cases}\frac{\mu(1+\alpha \beta)}{\theta-p \mu(1+\alpha \beta)}, & \rho<1, \\ \infty, & \rho \geq 1,\end{cases}
$$

where $\rho=p \mu(1+\alpha \beta) / \theta$ is the traffic intensity of the considered queueing system.

The server busy period initiated with $i$ customers is denoted by $b^{\langle i\rangle}$. Then, $b^{\langle i\rangle}$ can be expressed as $b^{\langle i\rangle}=\zeta_{1}+\cdots+\zeta_{i}$, where $\zeta_{1}, \ldots, \zeta_{i}$ are independent of each other and have the same distribution as $b$. Thus the PGF of $b^{\langle i\rangle}$ is $b^{i}(z)$.

Definition 7. The "system idle period" denotes the time interval from the time the system becomes empty until the first customer arrives and enters the system.

Definition 8. The "server idle period" denotes the time interval from the time the system becomes empty until the server begins to serve the first customer.

Let $I_{j}, l_{j}$, and $b_{j}$ denote the $j$ th system idle period, server idle period, and server busy period initiated with the initial state $N\left(0^{+}\right)(\geq 0)$, respectively; then, from the system assumptions, $\left\{I_{j}, j \geq 1\right\},\left\{l_{j}, j \geq 1\right\}$, and $\left\{b_{j}, j \geq 1\right\}$ are independent random variables, respectively. Further, $\left\{I_{k}, k \geq\right.$ $1\}$ follow an identical geometric distribution with mean $1 / p$. For $\left\{l_{j}, j \geq 1\right\}$ and $\left\{b_{j}, j \geq 1\right\}$, the following is observed from assumption (5).

(i) If $N\left(0^{+}\right)=0$, then

$$
\begin{aligned}
& l_{j}= \begin{cases}I_{1}, & j=1, \\
I_{j}+\tau_{1}+\cdots+\tau_{N-1}, & j \geq 2,\end{cases} \\
& b_{j}= \begin{cases}b, & j=1, \\
b^{\langle N\rangle}, & j \geq 2 .\end{cases}
\end{aligned}
$$

In this case, for $|z|<1$, the PGFs of $l_{j}, j \geq 1$ and $b_{j}, j \geq 1$ are given, respectively, by

$$
\begin{aligned}
& \sum_{k=1}^{\infty} P\left(l_{j}=k\right) z^{k}= \begin{cases}f(z), & j=1, \\
f^{N}(z), & j \geq 2,\end{cases} \\
& \sum_{k=1}^{\infty} P\left(b_{j}=k\right) z^{k}= \begin{cases}b(z), & j=1, \\
b^{N}(z), & j \geq 2,\end{cases}
\end{aligned}
$$

where $f(z)=p z /(1-\bar{p} z),|z|<1, \bar{p}=1-p$.

(ii) If $N\left(0^{+}\right)>0$, then $l_{j}=I_{j}+\tau_{1}+\cdots+\tau_{N-1}$ and $b_{j}=b^{\langle N\rangle}$, $j \geq 1$. Thus, the PGFs of $l_{j}$ and $b_{j} j \geq 1$ are $f^{N}(z)$ and $b^{N}(z)$, $|z|<1$, respectively.

Lemma 9 (see [19]). If $|z|<1, c_{n}>0$, and $c(z)=\sum_{n=0}^{\infty} c_{n} z^{n}$, then

$$
\lim _{z \uparrow 1}(1-z) c(z)=c<\infty \Longleftrightarrow \lim _{n \rightarrow \infty} \frac{1}{n} \sum_{k=1}^{n} c_{k}=c<\infty .
$$

Lemma 10 (see [19]). If $\lim _{n \rightarrow \infty} c_{n}=c<\infty$, then $\lim _{n \rightarrow \infty}(1 / n) \sum_{k=1}^{n} c_{k}=c$.

\section{Analysis of System Size Distribution}

In this section, above all, we will discuss the system size distribution at any epoch $n^{+}$in server busy period $b$. Next, the PGF of transient system size distribution at any epochs $n^{+}$is obtained. Finally, the steady-state system size distribution is derived.

3.1. Transient System Size Distribution in Server Busy Periodb. Let $Q_{j}\left(n^{+}\right)=P\left\{b>n^{+}, N\left(n^{+}\right)=j\right\}, j \geq 1$, denote transient system size distribution at epoch $n^{+}$in server busy period $b$ and $q_{j}^{+}(z)=\sum_{n=0}^{\infty} Q_{j}\left(n^{+}\right) z^{n},|z|<1$; then Theorem 11 follows. 
Theorem 11. For $j \geq 1$ and $|z|<1$,

$$
\begin{aligned}
& q_{j}^{+}(z)=\frac{1}{\widehat{G}(\bar{p} z)} \\
& \times\left\{\sum_{n=j-1}^{\infty} z^{n} C_{n}^{j-1} p^{j-1} \bar{p}^{n-j+1} \sum_{k=n+1}^{\infty} \widehat{g}_{k}+\sum_{i=1}^{j-1} \frac{q_{j-i}^{+}(z)}{b^{i+1}(z)}\right. \\
& \times\left[\widehat{G}(\bar{p} z+p z b(z))-\sum_{k=1}^{i} \widehat{g}_{k}(\bar{p} z+p z b(z))^{k}\right. \\
& \left.\left.-\sum_{k=i+1}^{\infty} \widehat{g}_{k} z^{k} \sum_{r=0}^{i} C_{k}^{r}(p b(z))^{r} \bar{p}^{k-r}\right]\right\},
\end{aligned}
$$

where $\bar{p}=1-p$ and $\sum_{i=1}^{j}=0$ if $j \leq 0$.

Proof. Denote by $\widehat{\chi}_{1}$ the total generalized service time of the first customer served in server busy period $b$, and $v$ represents the arriving number of customers during $\widehat{\chi}_{1}$. Noting that the length of $b$ has nothing to do with the service order of customers and arrival interval follows a geometric distribution with parameter $p$, so we get

$$
P(v=j)=\sum_{k=j}^{\infty} \widehat{g}_{k} C_{k}^{j} p^{j} \bar{p}^{k-j}, \quad j \geq 0 .
$$

Let $A_{1}, A_{2}, \ldots, A_{v}$ be the customers who arrive in the system during $\widehat{\chi}_{1}$, called class- 1 customers. We call those who arrive after the class- 1 customers as class- 2 customers. Since the length of server busy period is irrelevant to the service order of customers, we adopt the following service order: class- 1 customers are served in the order of $A_{1}, A_{2}, \ldots, A_{v}$. After serving each class-1 customer, the server will serve any class- 2 customer until there are no class- 2 customers present. Thus the server busy period $b$ can be decomposed as

$$
b=\widehat{\chi}_{1}+L_{1}+\cdots+L_{v}
$$

where $L_{k}, 1 \leq k \leq v$, denotes the time interval from the epoch when the server begins to serve the $k$ th class- 1 customer until the next epoch when the service of the $(k+1)$ th class- 1 customer begins. Therefore, $L_{k}(k=1,2, \ldots, v)$ are independent random variables with the same distribution as b. Obviously, $L_{1}+L_{2}+\cdots+L_{v}=0$ when $v=0$. Since the point when the server busy period ends is a renewal point, for $j \geq 1, Q_{j}\left(n^{+}\right)$can be expressed as

$$
\begin{aligned}
Q_{j}\left(n^{+}\right)= & P\left\{\widehat{\chi}_{1}+L_{1}+\cdots+L_{v}>n^{+}, N\left(n^{+}\right)=j\right\} \\
= & P\left\{\widehat{\chi}_{1}>n^{+}, N\left(n^{+}\right)=j\right\} \\
& +P\left\{\widehat{\chi}_{1} \leq n^{+}<\widehat{\chi}_{1}+L_{1}+\cdots+L_{v}, N\left(n^{+}\right)=j\right\} \\
= & P\left\{\widehat{\chi}_{1}>n^{+}, j-1 \text { customers arrive in }\left(0, n^{+}\right]\right\} \\
& +\sum_{k=1}^{n} \widehat{g}_{k} \sum_{i=0}^{k} C_{k}^{i} p^{i} \bar{p}^{k-i} \\
& \times P\left\{L_{1}+\cdots+L_{i}>(n-k)^{+},\right. \\
& \left.\quad N\left((n-k)^{+}\right)=j\right\} .
\end{aligned}
$$

In the light of the above service order, if the epoch $(n-k)^{+}$ is in $L_{m}(1 \leq m \leq i)$ and $N\left((n-k)^{+}\right)=j$, then at epoch $(n-k)^{+}$there are $i-m$ class-1 customers waiting for service and $j-(i-m)$ class -2 customers present in the system. By the law of total probability and noting that $L_{m}(1 \leq m \leq i)$ has the same probability property as $b$, we get

$$
\begin{aligned}
& P\left\{L_{1}+\cdots+L_{i}>(n-k)^{+}, N\left((n-k)^{+}\right)=j\right\} \\
& =\sum_{m=1}^{i} P\left\{L_{1}+\cdots+L_{m-1} \leq(n-k)^{+}<L_{1}+\cdots+L_{m},\right. \\
& \left.N\left((n-k)^{+}\right)=j\right\} \\
& =\sum_{m=1}^{i} \sum_{l=m-1}^{n-k} P\left\{L_{1}+\cdots+L_{m-1}=l\right\} \\
& \quad \times P\left\{L_{m}>(n-k-l)^{+},\right. \\
& =\sum_{m=1}^{i} \sum_{l=m-1}^{n-k} P\left\{L_{1}+\cdots+L_{m-1}=l\right\} Q_{j-i+m}\left((n-k-l)^{+}\right) .
\end{aligned}
$$

Substituting (11) into (10) leads to

$$
\begin{aligned}
Q_{j}\left(n^{+}\right)= & \sum_{k=n+1}^{\infty} \widehat{g}_{k} C_{n}^{j-1} p^{j-1} \bar{p}^{n-j+1} \\
& +\sum_{k=1}^{n} \widehat{g}_{k} \sum_{i=0}^{k} C_{k}^{i} p^{i} \bar{p}^{k-i} \\
& \times \sum_{m=1}^{i} \sum_{l=m-1}^{n-k} P\left\{L_{1}+\cdots+L_{m-1}=l\right\} \\
& \times Q_{j-i+m}\left((n-k-l)^{+}\right) .
\end{aligned}
$$

Taking the PGF of (12) completes the proof of Theorem 11. 
3.2. Transient System Size Distribution at Any Epoch $n^{+}$. Let $P_{i j}\left(n^{+}\right)=P\left\{N\left(n^{+}\right)=j \mid N\left(0^{+}\right)=i\right\}, i, j \geq 0$, be conditional distribution of transient system size at any epochs $n^{+}$and $p_{i j}(z)=\sum_{n=0}^{\infty} P_{i j}\left(n^{+}\right) z^{n},|z|<1$; then Theorem 12 follows.

Theorem 12. For $|z|<1$ and $i \geq 1$,

$$
\begin{aligned}
& p_{00}(z)=\frac{1-f(z)}{1-z}\left\{1+\frac{f(z) b(z)}{1-[f(z) b(z)]^{N}}\right\}, \\
& p_{i 0}(z)=\frac{[1-f(z)] b^{i}(z)}{(1-z)\left\{1-[f(z) b(z)]^{N}\right\}},
\end{aligned}
$$

where $f(z)=p z /(1-\bar{p} z)$ and $b(z)$ is determined by Lemma 6 .

Proof. Noting that the system is empty at time epoch $n^{+}$if and only if the system is in the "system idle period," thus we have

$$
\begin{aligned}
P_{00}\left(n^{+}\right)= & P\left\{0 \leq n^{+}<I_{1}\right\} \\
& +\sum_{k=2}^{\infty} P\left\{\sum_{i=1}^{k-1}\left(l_{i}+b_{i}\right) \leq n^{+}<\sum_{i=1}^{k-1}\left(l_{i}+b_{i}\right)+I_{k}\right\} \\
= & P\left\{I_{1}>n^{+}\right\} \\
& +\sum_{k=2}^{\infty} \sum_{u=2 k-2}^{n} P\left\{\sum_{i=1}^{k-1}\left(l_{i}+b_{i}\right)=u\right\} \\
& \times P\left\{I_{k}>(n-u)^{+}\right\}
\end{aligned}
$$

where $I_{i}, l_{i}$, and $b_{i}$ denote the $i$ th "system idle period," the $i$ th "server idle period," and the $i$ th "server busy period," respectively (see Definitions 5-8).

For $i \geq 1$, similarly, we have

$$
\begin{aligned}
P_{i 0}\left(n^{+}\right)= & \sum_{u=i}^{n} P\left\{b^{\langle i\rangle}=u\right\} P\left\{I_{1}>(n-u)^{+}\right\} \\
& +\sum_{k=3}^{\infty} \sum_{u=2 k+i-4}^{n} P\left\{\sum_{i=1}^{k-2}\left(l_{i}+b_{i}+b^{\langle i\rangle}\right)=u\right\} \\
& \times P\left\{I_{k-1}>(n-u)^{+}\right\} .
\end{aligned}
$$

Taking the PGFs of (14) and (15), respectively, and using the PGFs of $I_{i}, l_{i}, b_{i}$, and $b^{\langle i\rangle}$, we can obtain (13).
Theorem 13. For $|z|<1, i \geq 1$, and $j=1,2, \ldots, N-1$,

$$
\begin{aligned}
p_{0 j}(z)= & f(z) q_{j}^{+}(z) \\
& +\left(\frac{1-f(z)}{1-z} f^{j+1}(z) b(z)\right. \\
& \left.\quad+f^{N+1}(z) b(z) \sum_{k=1}^{N} q_{j-N+k}(z) b^{k-1}(z)\right) \\
& \times\left(1-[f(z) b(z)]^{N}\right)^{-1}, \\
p_{i j}(z)=\sum_{k=1}^{i} q_{j-i+k}^{+}(z) b^{k-1}(z) & \\
+ & \left(\frac{1-f(z)}{1-z} f^{j}(z) b^{i}(z)\right. \\
& \left.\quad+f^{N}(z) b^{i}(z) \sum_{k=1}^{N} q_{j-N+k}(z) b^{k-1}(z)\right) \\
& \left.\times(f(z) b(z)]^{N}\right)^{-1},
\end{aligned}
$$

where $f(z)=p z /(1-\bar{p} z)$ and $b(z)$ and $q_{j}^{+}(z)$ are determined by Lemma 6 and Theorem 11, respectively.

Proof. For $j=1,2, \ldots, N-1, N\left(n^{+}\right)=j$ means that epoch $n^{+}$is in server busy period or server idle period with some customers waiting for service, so

$$
\begin{aligned}
P_{0 j}\left(n^{+}\right)= & P\left\{I_{1} \leq n^{+}<I_{1}+b_{1}, N\left(n^{+}\right)=j\right\} \\
& +P\left\{I_{1}+b_{1}+I_{2}+\sum_{l=1}^{j-1} \tau_{l}\right. \\
& \left.\leq n^{+}<I_{1}+b_{1}+I_{2}+\sum_{l=1}^{j} \tau_{l}\right\} \\
& +P\left\{I_{1}+b_{1}+I_{2}+\sum_{l=1}^{N-1} \tau_{l} \leq n^{+}, N\left(n^{+}\right)=j\right\} \\
= & \sum_{u=1}^{n} P\left\{I_{1}=u\right\} Q_{j}\left((n-u)^{+}\right) \\
& +\sum_{u=j+2}^{n} P\left\{I_{1}+b_{1}+I_{2}+\sum_{l=1}^{j-1} \tau_{l}=u\right\} \\
& \times P\left\{\tau_{j}>(n-u)^{+}\right\} \\
& +\sum_{u=N+2}^{n} P\left\{I_{1}+b_{1}+I_{2}+\sum_{l=1}^{N-1} \tau_{l}=u\right\} \\
& \times P_{N j}\left((n-u)^{+}\right) .
\end{aligned}
$$


For $i \geq 1$, similarly, we get

$$
\begin{aligned}
P_{i j}\left(n^{+}\right)= & \sum_{k=1}^{i} \sum_{u=k-1}^{n} P\left\{b^{\langle k-1\rangle}=u\right\} \\
& \times Q_{j-i+k}\left((n-u)^{+}\right) \\
& +\sum_{u=j+i}^{n} P\left\{b^{\langle i\rangle}+I_{2}+\sum_{l=1}^{j-1} \tau_{l}=u\right\} \\
& \times P\left\{\tau_{j}>(n-u)^{+}\right\} \\
& +\sum_{u=N+i}^{n} P\left\{b^{\langle i\rangle}+I_{2}+\sum_{l=1}^{N-1} \tau_{l}=u\right\} \\
& \times P_{N j}\left((n-u)^{+}\right) .
\end{aligned}
$$

Taking the PGFs of (18) and (19), respectively, gives rise to

$$
\begin{aligned}
p_{0 j}(z)= & f(z) q_{j}^{+}(z) \\
& +\frac{1-f(z)}{1-z} f^{j+1}(z) b(z)+f^{N+1}(z) b(z) p_{N j}(z), \\
p_{i j}(z)= & \sum_{k=1}^{i} q_{j-i+k}^{+}(z) b^{k-1}(z) \\
& +\frac{1-f(z)}{1-z} f^{j}(z) b^{i}(z)+f^{N}(z) b^{i}(z) p_{N j}(z),
\end{aligned}
$$

which leads to the following relationship between $p_{i j}(z)$ and $p_{0 j}(z)$ :

$$
\begin{aligned}
p_{i j}(z)= & \sum_{k=1}^{i} q_{j-i+k}^{+}(z) b^{k-1}(z) \\
& +\frac{b^{i-1}(z)}{f(z)} p_{0 j}(z)-b^{i-1}(z) q_{j}^{+}(z), \\
& \quad i=1,2, \ldots, \quad j=1,2, \ldots, N-1 .
\end{aligned}
$$

Substituting (22) into (20) yields (16), and, furthermore, we obtain (17).

Theorem 14. For $|z|<1, i \geq 1$, and $j=N, N+1, N+2, \ldots$,

$$
\begin{aligned}
p_{0 j}(z)= & f(z) q_{j}^{+}(z) \\
& +\frac{f^{N+1}(z) b(z) \sum_{k=1}^{N} q_{j-N+k}^{+}(z) b^{k-1}(z)}{1-[f(z) b(z)]^{N}}, \\
p_{i j}(z)= & \sum_{k=1}^{i} q_{j-i+k}^{+}(z) b^{k-1}(z) \\
& +\frac{f^{N}(z) b^{i}(z) \sum_{k=1}^{N} q_{j-N+k}^{+}(z) b^{k-1}(z)}{1-[f(z) b(z)]^{N}},
\end{aligned}
$$

where $f(z)=p z /(1-\bar{p} z)$ and $b(z)$ and $q_{j}^{+}(z)$ are determined by Lemma 6 and Theorem 11, respectively.

Proof. For $j=N, N+1, N+2, \ldots, N\left(n^{+}\right)=j$ indicates that epoch $n^{+}$is in server busy period, so we have

$$
\begin{aligned}
& P_{0 j}\left(n^{+}\right)=\sum_{u=1}^{n} P\left\{I_{1}=u\right\} Q_{j}\left((n-u)^{+}\right) \\
&+\sum_{u=N+2}^{n} P\left\{I_{1}+b_{1}+I_{2}+\sum_{k=1}^{N-1} \tau_{k}\right\} \\
& \quad \times P_{N j}\left((n-u)^{+}\right), \\
& P_{i j}\left(n^{+}\right)= \sum_{k=1}^{i} Q_{j-i+k}\left((n-u)^{+}\right) \\
& \times \sum_{u=k-1}^{n} P\left\{b^{\langle k-1\rangle}=u\right\} \\
&+\sum_{u=N+i}^{n} P\left\{b^{\langle i\rangle}+I_{2}+\sum_{k=1}^{N-1} \tau_{k}=u\right\} \\
& \times P_{N j}\left((n-u)^{+}\right), \quad i \geq 1 .
\end{aligned}
$$

Taking PGFs of (24) and performing similar operations in the proof of Theorem 13, we can complete the proof.

\subsection{Steady-State System Size Distribution at Any Epoch $n^{+}$}

Theorem 15. Letting $p_{j}^{+}=\lim _{n \rightarrow \infty} P\left\{N\left(n^{+}\right)=j\right\}, j=$ $0,1,2, \ldots$, then

(1) for $\rho=p \mu(1+\alpha \beta) / \theta \geq 1, p_{j}^{+}=0, j=0,1,2, \ldots$;

(2) for $\rho=p \mu(1+\alpha \beta) / \theta<1$, the steady-state system size distribution $\left\{p_{j}^{+}, j=0,1,2, \ldots\right\}$ is given by the following formulas:

$$
\begin{aligned}
& p_{0}^{+}=\frac{1-\rho}{N} \\
& p_{j}^{+}=\frac{1-\rho}{N}\left(1+p \sum_{k=1}^{N} \Delta_{j-N+k}\right), \quad j=1,2, \ldots, N-1, \\
& p_{j}^{+}=\frac{1-\rho}{N} p \sum_{k=1}^{N} \Delta_{j-N+k}, \quad j=N, N+1, \ldots,
\end{aligned}
$$


(3) where

$$
\begin{gathered}
\Delta_{1}=\frac{1-\widehat{G}(\bar{p})}{p \widehat{G}(\bar{p})}, \\
\Delta_{j}=\frac{1}{\widehat{G}(\bar{p})}\left\{\sum_{n=j-1}^{\infty} C_{n}^{j-1} p^{j-1} \bar{p}^{n-j+1} \sum_{k=n+1}^{\infty} \widehat{g}_{k}\right. \\
\left.+\sum_{i=1}^{j-1} \Delta_{j-i}\left[1-\sum_{k=1}^{i} \widehat{g}_{k}-\sum_{k=i+1}^{\infty} \widehat{g}_{k} \sum_{r=0}^{i} C_{k}^{r} p^{r} \bar{p}^{k-r}\right]\right\}, \\
j=2,3, \ldots,
\end{gathered}
$$

(4) when $j \leq 0, \sum_{i=1}^{j}=0$.

Proof. Applying $0 \leq P_{i j}\left(n^{+}\right) \cdot P\left\{N\left(0^{+}\right)=i\right\}<1$ and Lemmas 9 and 10 yields

$$
\begin{aligned}
p_{j}^{+} & =\lim _{n \rightarrow \infty} \sum_{i=0}^{\infty} P_{i j}\left(n^{+}\right) P\left\{N\left(0^{+}\right)=i\right\} \\
& \left.=\sum_{i=0}^{\infty} P\left\{N\left(0^{+}\right)=i\right\} \lim _{n \rightarrow \infty} P_{i j}\left(n^{+}\right)=\lim _{z \uparrow 1}(1-z) p_{i j}(z) .\right]
\end{aligned}
$$

So $\left\{p_{j}^{+}, j \geq 0\right\}$ is obtained by Lemma 6, Theorems 11-14, and L'Hospital's rule.

Also, for $\rho=p \mu(1+\alpha \beta) / \theta<1, \sum_{j=0}^{\infty} p_{j}^{+}=1$ is clear only by noting that

$$
\begin{aligned}
\sum_{j=1}^{\infty} z^{j} \sum_{k=1}^{N} \Delta_{j-N+k} & =\frac{z\left(1-z^{N}\right)[1-\widehat{G}(\bar{p}+p z)]}{p(1-z)[\widehat{G}(\bar{p}+p z)-z]}, \\
\sum_{j=1}^{\infty} \sum_{k=1}^{N} \Delta_{j-N+k} & =\frac{N \rho}{p(1-\rho)} .
\end{aligned}
$$

Corollary 16. Let $\pi^{+}(z)=\sum_{j=0}^{\infty} p_{j}^{+} z^{j},|z|<1$, denote the PGF of steady-state system size distribution $\left\{p_{j}^{+}, j \geq 0\right\}$ at any epoch $n^{+}$; then, for $\rho=p \mu(1+\alpha \beta) / \theta<1$,

$$
\pi^{+}(z)=\frac{(1-\rho)(1-z) \widehat{G}(\bar{p}+p z)}{\widehat{G}(\bar{p}+p z)-z} \cdot \frac{1-z^{N}}{(1-z) N} .
$$

Proof. Using $\pi^{+}(z)=p_{0}^{+}+\sum_{j=1}^{\infty} p_{j}^{+} z^{j}$ and (25), we obtain (29).

Remark 17. Equation (29) means that the system size $L^{+}$of $\mathrm{N}$ policy Geo/G/1 queue with feedback and repairable server is the sum of two independent random variables: $L^{+}=L_{0}^{+}+L_{1}^{+}$, if $\rho=p \mu(1+\alpha \beta) / \theta<1 . L_{0}^{+}$is the system size of $\mathrm{Geo} / \mathrm{G} / 1$ queue with feedback and repairable server, and $L_{1}^{+}$is the additional system size due to the $\mathrm{N}$-policy. This confirms that the stochastic decomposition property by Fuhrmann and Cooper [20] is also valid for the discrete-time queue under consideration.

Corollary 18. Let $E\left[L^{+}\right]$denote the expected steady-state system size at any epoch $n^{+}$; then, for $\rho=p \mu(1+\alpha \beta) / \theta<1$,

$$
E\left[L^{+}\right]=\rho+\frac{p^{2} \widehat{G}^{\prime \prime}(1)}{2(1-\rho)}+\frac{N-1}{2} .
$$

Proof. Equation (30) is easily obtained by $E\left[L^{+}\right]=$ $\left.\left(d \pi^{+}(z) / d z\right)\right|_{z=1}$.

Remark 19 (special case). If $\theta \rightarrow 1$ and $\alpha \rightarrow 0$ (no feedback and no server breakdown), then our model becomes a discrete-time $\mathrm{N}$-policy Geo/G/1 queueing system. In this case, for $\rho=p \mu<1$, we have

$$
\begin{aligned}
& \pi^{+}(z)=\frac{(1-\rho)(1-z) G(\bar{p}+p z)}{G(\bar{p}+p z)-z} \cdot \frac{1-z^{N}}{(1-z) N}, \\
& E\left[L^{+}\right]=\rho+\frac{p^{2} G^{\prime \prime}(1)}{2(1-\rho)}+\frac{N-1}{2},
\end{aligned}
$$

which agree with those in [8] (setting $p=1$ in (15), (16), and (18) for [8]'s model).

\section{A Numerical Example}

Our study has a potential application in a network access proxy system. In such a system, Channel requests, grants, data transmissions, and receptions all proceed in fixed time intervals. That is, service request sending, preprocessing, and processing are done in a discrete-time manner. Service requests sent by the users can be modelled as a Bernoulli process with rate $p$. To avoid frequent switchovers from idle state to busy state, the proxy server is designed to start serving exhaustively, while the service requests are accumulated to $N$. If a service request is received with errors at the destination, it is resent (feedback) with probability $1-\theta$. When the server is busy, the arriving requests are placed in queue. The service time of each request is assumed to follow a geometric distribution with mean $\mu$. Also, the service may be interrupted (server breakdown) due to some unpredictable events, such as traffic congestion of the network. Suppose that unpredictable events occur according to a Bernoulli process with rate $\alpha$ and service interruption is immediately recovered. The recovery time obeys a geometric distribution with mean $\beta$. The service will continuously start when the interruption is recovered.

Numerical results in Table 1 illustrate the application of the steady-state system size distribution in system capacity design. Here, we let $p=0.15, \mu=2, \theta=0.9, \alpha=0.01, \beta=5$, and $N=5$. By Theorem 15 and Corollary 18, we numerically obtain the steady-state service request number distribution $\left\{p_{j}^{+}, j \geq 0\right\}$, the mean service request number $E\left(L^{+}\right)$, and the system load $\rho$ for the above network access proxy system (see Table 1). 
TABLE 1: The steady-state service request number distribution for a network access proxy system $(p=0.15, \mu=2, \theta=0.9, \alpha=0.01, \beta=5$, and $N=5$ ).

\begin{tabular}{lccccccc}
\hline$p_{0}^{+}$ & $p_{1}^{+}$ & $p_{2}^{+}$ & $p_{3}^{+}$ & $p_{4}^{+}$ & $p_{5}^{+}$ & $p_{6}^{+}$ & $p_{7}^{+}$ \\
0.08333 & 0.14661 & 0.17341 & 0.18040 & 0.18265 & 0.10049 & 0.03816 & $p_{14}^{+}$ \\
\hline$p_{8}^{+}$ & $p_{9}^{+}$ & $p_{10}^{+}$ & $p_{11}^{+}$ & $p_{12}^{+}$ & $p_{13}^{+}$ & 0.01227 \\
0.00618 & 0.00483 & 0.00456 & 0.00452 & 0.00427 & 0.00388 & 0.00336 & 0.00276 \\
\hline$p_{16}^{+}$ & $p_{17}^{+}$ & $p_{18}^{+}$ & $p_{19}^{+}$ & $p_{20}^{+}$ & $p_{21}^{+}$ & $p_{22}^{+}$ & 0.00050 \\
0.00210 & 0.00162 & 0.00127 & 0.00100 & 0.00079 & 0.00063 & $p_{23}^{+}$ \\
\hline$p_{24}^{+}$ & $p_{25}^{+}$ & $p_{26}^{+}$ & $p_{27}^{+}$ & $p_{28}^{+}$ & $p_{29}^{+}$ & 0.00040 \\
0.00032 & 0.00026 & 0.00021 & 0.00016 & 0.00013 & 0.00010 & 0.00008 \\
\hline$p_{32}^{+}$ & $p_{33}^{+}$ & $p_{34}^{+}$ & $p_{35}^{+}$ & $p_{36}^{+}$ & $p_{37}^{+}$ & 0.00007 \\
0.00005 & 0.00004 & 0.00003 & 0.00002 & 0.00001 & 0.00000 & $p_{38}^{+}$ & 0.00000 \\
\hline \multicolumn{2}{c}{$E\left(L^{+}\right)=3.11583, \rho=0.58333<1, \sum_{j=0}^{39} p_{j}^{+}=0.96461$} & $p_{39}^{+}$ \\
\hline
\end{tabular}

With Matlab 7.0, the data here are accurate for five places of decimals.

TABLE 2: The optimal system capacity of a network access proxy system for different values of controllable parameter $\gamma(p=0.15, \mu=2$, $\theta=0.9, \alpha=0.01, \beta=5$, and $N=5$ ).

\begin{tabular}{lcccccc}
\hline$\gamma$ & 0.00003 & 0.00015 & 0.00250 & 0.00615 & 0.01725 & 0.04550 \\
$M^{*}$ & 34 & 31 & 24 & 18 & 13 & 7 \\
\hline
\end{tabular}

Denote by $L^{+}$the service request number in the system. According to Table 1, the probabilities that $L^{+}$exceeds $E\left(L^{+}\right)-$ 1, $E\left(L^{+}\right)$, and $E\left(L^{+}\right)+1$ are obtained as

$$
\begin{aligned}
P\left\{L^{+}>E\left(L^{+}\right)-1\right\} & =1-P\left\{L^{+} \leq E\left(L^{+}\right)-1\right\} \\
& =1-\sum_{j=0}^{2} p_{j}^{+}=0.59664, \\
P\left\{L^{+}>E\left(L^{+}\right)\right\} & =1-P\left\{L^{+} \leq E\left(L^{+}\right)\right\} \\
& =1-\sum_{j=0}^{3} p_{j}^{+}=0.41624, \\
P\left\{L^{+}>E\left(L^{+}\right)+1\right\} & =1-P\left\{L^{+} \leq E\left(L^{+}\right)+1\right\} \\
& =1-\sum_{j=0}^{4} p_{j}^{+}=0.23358 .
\end{aligned}
$$

The above three probability values are greater than $23 \%$, which shows that it is quite inaccurate for system capacity design to use mean service request number $E\left(L^{+}\right)$because quite a few service requests will be lost due to no waiting space. Also, it is seen from Table 1 that the probability that the service request number in the system exceeds 29 is less than $0.01 \%$. So it is also unnecessary for this system to design a large capacity.

Let $M$ be system capacity and let the positive decimal $\gamma$ be less than 1 . For simplicity, we assume that system capacity $M$ takes positive integer value. When the overflow probability $P\left(L^{+}>M\right) \leq \gamma$, from Table 1 , we can get optimal system capacity $M^{*}$ so that system operating costs are minimized (see Table 2). For example, if $\gamma=0.00015$, then it follows from $P\left(L^{+}>M\right) \leq \gamma$ and Table 1 that $M \geq 31$. Thus we take $M^{*}=31$ since small capacity leads to small system cost.

\section{Conclusions}

In this paper, we consider a discrete-time N-policy Geo/G/1 queueing system with feedback and repairable server. With a probability analysis and renewal process theory, we discuss the transient system size distribution and derive the steadystate system size distribution and its PGF. It should be noted that the steady-state system size distribution derived by this paper is quite suitable for numerical calculation. Numerical examples show the application of steady-state system size distribution in system capacity design for a network access proxy system. In the future, further study, such as the random N-policy Geo ${ }^{X} / \mathrm{G} / 1$ queueing system with feedback and repairable server, will be the research topic with the help of similar ideas and methods.

\section{Conflict of Interests}

The authors declare that there is no conflict of interests regarding the publication of this paper.

\section{Acknowledgments}

The authors would like to thank the referees and the editor for their valuable comments and suggestions. This work is supported by the Basic and Frontier Research Foundation of Chongqing of China (cstc2013jcyjA00008) and the Scientific Research Starting Foundation for Doctors of Chongqing University of Technology (2012ZD48).

\section{References}

[1] H. Kobayashi and A. G. Konheim, "Queueing models for computer communications system analysis," IEEE Transactions on Communications, vol. 25, no. 1, pp. 2-29, 1977. 
[2] I. Rubin and L. F. M. De Moraes, "Message delay analysis for polling and token multiple-access schemes for local communication networks," IEEE Journal on Selected Areas in Communications, vol. 1, no. 5, pp. 935-947, 1983.

[3] F. J. Li and R. H. Sun, "Measurement-estimation approach to efficiency evaluation of bandwidth allocation scheme in ATM networks," in Proceedings of the Conference Record IEEE Internation Conference on Conmunications, vol. 6, no. 2, pp. 131142, 1997.

[4] N. S. Tian, X. L. Xu, and Z. Y. Ma, Discrete-Time Queueing Theory, Science Press, Beijing, China, 2008.

[5] M. Yadin and P. Naor, "Queueing systems with a removable service station," Operational Research Quarterly, vol. 14, no. 3, pp. 393-405, 1963.

[6] L. Tadj and G. Choudhury, "Optimal design and control of queues," Top, vol. 13, no. 2, pp. 359-412, 2005.

[7] P. Moreno, "A discrete-time single-server queue with a modified $N$-policy," International Journal of Systems Science, vol. 38, no. 6, pp. 483-492, 2007.

[8] T.-Y. Wang and J.-C. Ke, "The randomized threshold for the discrete-time Geo/G/1 queue," Applied Mathematical Modelling, vol. 33, no. 7, pp. 3178-3185, 2009.

[9] Y. Y. Wei, M. M. Yu, Y. H. Tang, and J. X. Gu, "Queue size distribution and capacity optimum design for $N$-policy $G_{e o}{ }^{\left(\lambda_{1}, \lambda_{2}, \lambda_{3}\right)} / G / 1$ queue with setup time and variable input rate," Mathematical and Computer Modelling, vol. 57, no. 5-6, pp. 1559-1571, 2013.

[10] T.-Y. Wang, "Random $N$-policy Geo/G/1 queue with startup and closedown times," Journal of Applied Mathematics, vol. 2012, Article ID 793801, 19 pages, 2012.

[11] L. Takács, "A single-server queue with feedback," The Bell System Technical Journal, vol. 42, no. 2, pp. 505-519, 1963.

[12] B. D. Choi, B. Kim, and S. H. Choi, "On the $M / G / 1$ Bernoulli feedback queue with multi-class customers," Computers \& Operations Research, vol. 27, no. 3, pp. 269-286, 2000.

[13] D. I. Choi and T.-S. Kim, "Analysis of a two-phase queueing system with vacations and Bernoulli feedback," Stochastic Analysis and Applications, vol. 21, no. 5, pp. 1009-1019, 2003.

[14] B. Krishna Kumar and J. Raja, "On multiserver feedback retrial queues with balking and control retrial rate," Annals of Operations Research, vol. 141, pp. 211-232, 2006.

[15] I. Atencia and P. Moreno, "Discrete-time $G e o^{[X]} / G_{H} / 1$ retrial queue with Bernoulli feedback," Computers \& Mathematics with Applications, vol. 47, no. 8-9, pp. 1273-1294, 2004.

[16] J. Wang and P. Zhang, "A discrete-time retrial queue with negative customers and unreliable server," Computers \& Industrial Engineering, vol. 56, no. 4, pp. 1216-1222, 2009.

[17] Y. H. Tang, X. Yun, and S. J. Huang, "Discrete-time $G e o^{X} / G / 1$ queue with unreliable server and multiple adaptive delayed vacations," Journal of Computational and Applied Mathematics, vol. 220, no. 1-2, pp. 439-455, 2008.

[18] Z. M. Liu and S. Gao, "Reliability indices of a Geo/G/1/1 Erlang loss system with active breakdowns under Bernoulli schedule," International Journal of Management Science and Engineering Management, vol. 5, no. 6, pp. 433-438, 2010.

[19] J. J. Hunter, Mathematical Techniques of Applied Probability, Discrete Time Models: Techniques and Applications, vol. 2 of Operations Research and Industrial Engineering, Academic Press, New York, NY, USA, 1983.

[20] S. W. Fuhrmann and R. B. Cooper, "Stochastic decompositions in the $M / G / 1$ queue with generalized vacations," Operations Research, vol. 33, no. 5, pp. 1117-1129, 1985. 


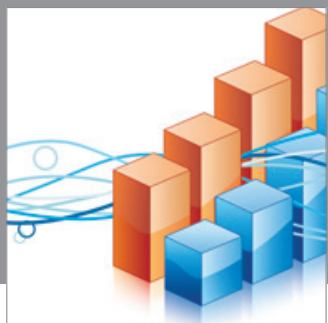

Advances in

Operations Research

mansans

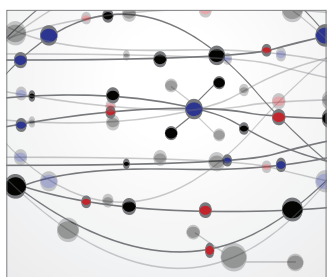

The Scientific World Journal
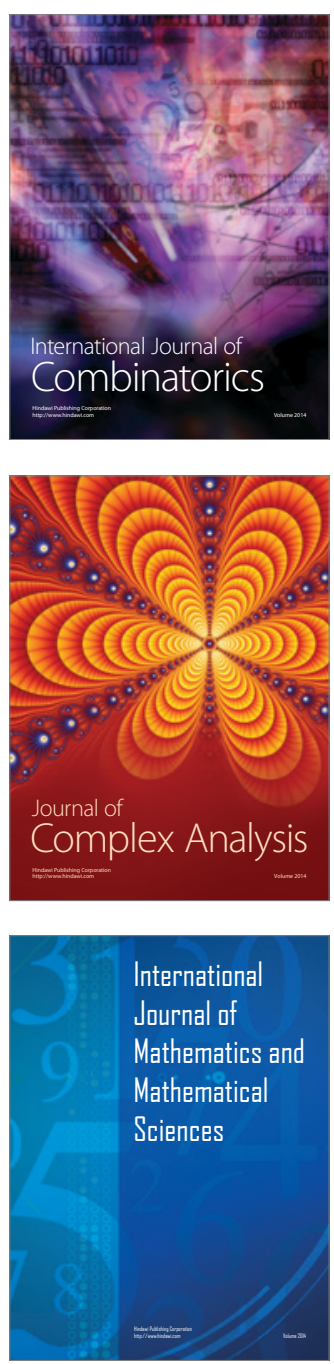
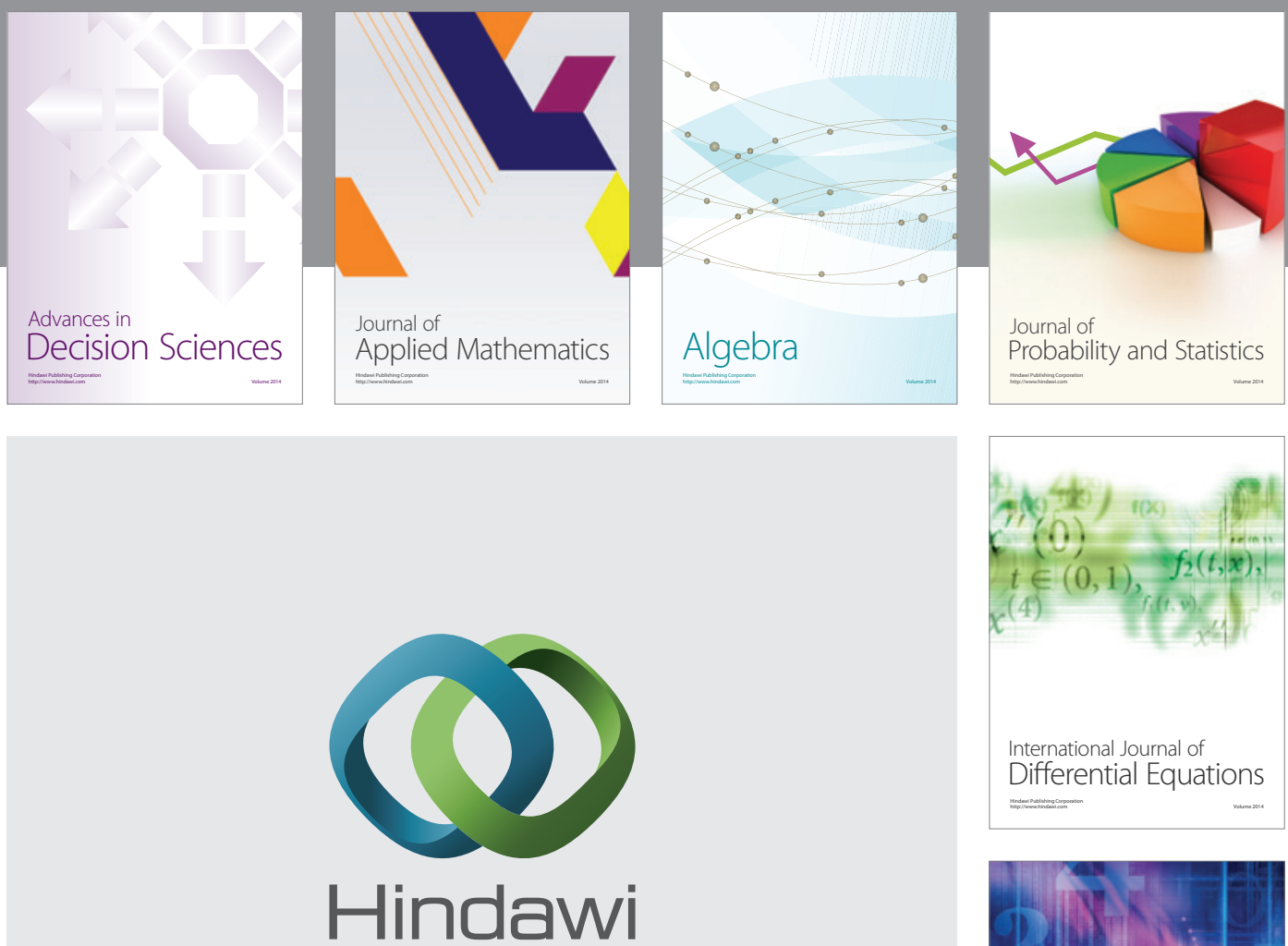

Submit your manuscripts at http://www.hindawi.com
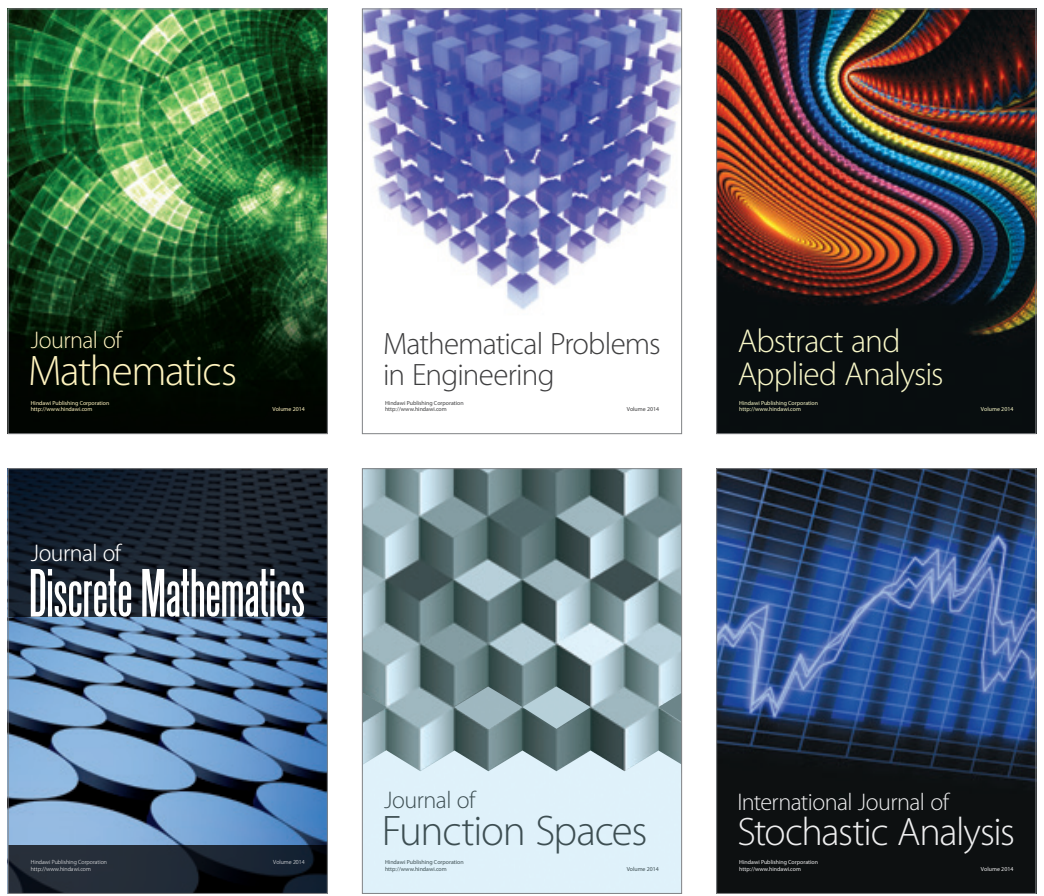

Journal of

Function Spaces

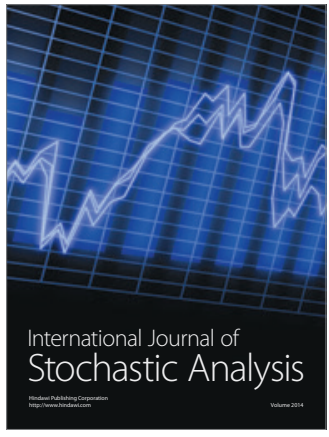

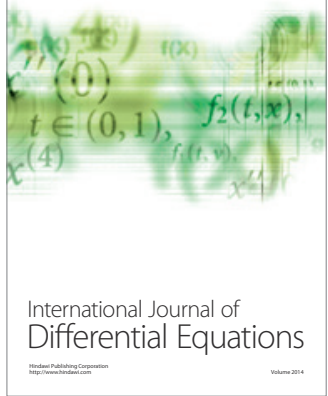
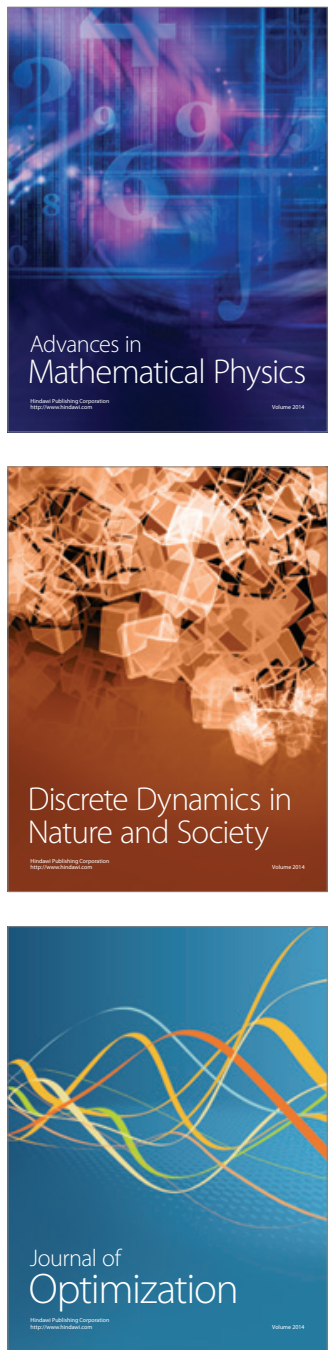